\title{
NOTE SUR LES MÉTHODES D'ESTIMATION DE L'ANGLE DE POITRINE DES POULETS DE GHAIR
}

\author{
F. H. RICARD \\ Domaine expérimental avicole du Magneraud, \\ St-Pierre d'Amilly (Charente-Maritime).
}

Les caractéristiques de conformation des poulets sont un élément important de leur classification commerciale. Pour satisfaire à la demande des éleveurs et aux exigences du marché, les poussins commerciaux utilisés par les producteurs de poulets doivent avoir une croissance et une conformation qui peuvent être génétiquement déterminées. I'angle de poitrine peut servir à mesurer, sur le poulet vivant, l'importance des masses musculaires pectorales, liées à la conformation thoracique. Nous proposons une méthode et un appareil susceptibles d'améliorer la précision de cette mesure et son utilisation dans la sélection.

\section{LES MÉTHODES D'ESTIMATION DE LA CONFORMATION THORACIQUE}

La première consiste à donner une appréciation subjective. Les spécialistes anglo-saxons utilisent depuis longtemps cette méthode pour classer les animaux selon les 3 catégories officielles $\mathrm{A}, \mathrm{B}$, et $\mathrm{C}$, qui sont à la base de leurs transactions commerciales. C'est une méthode peu précise et peu fidèle mais elle est rapide et à la portée de tous les éleveurs ayant 1'habitude de manipuler les animaux. Elle permet de repérer facilement les défauts dtı bréchet. Pour avoir des données valables, il faut tenir les poulets de la même façon et la même personne doit juger le lot entier.

La deuxième méthode consiste à mesurer la largeur des muscles de la poitrine à l'aide d'un pied à coulisse placé à un niveau donné. C'est une méthode plus précise mais moins rapide que l'appréciation subjective et elle risque d'être peu fidèle si on exerce une pression variable sur la poitrine de l'animal.

Une troisième méthode consiste à modeler sur la poitrine de l'animal un fil souple et malléable, tel que le fil pour soudure ou fusible électrique. On obtient ainsi la matérialisation du contour de la poitrine. On reporte ensuite le fil sur une feuille de papier où on peut mesurer soit la largeur à une distance donnée du sommet de la 
courbe, soit l'aire de la section comprise entre la courbe et la ligne correspondant à la largeur ci-dessus. Cette méthode a été utilisée dans de nombreuses études et il en existe de nombreuses variantes. La technique décrite par Asmuxdson, I944, est valable pour les poulets comme pour les dindons : l'animal est couché sur le dos, on modèle le fil dans un plan perpendiculaire à l'axe du corps et situé en arrière de l'extrémité du bréchet, on peut ensuite mesurer la largeur à différentes distances du sommet de la courbe. Certains auteurs ont remplacé le fil par un mince ruban de plomb. La méthode du fil ou du ruban est précise et fidèle, mais elle est longue à mettre en œuvre. Elle convient pour des travaux de recherche portant sur quelques animaux, mais non pour le sélectionneur qui doit manipuler des effectifs importants.

Une dernière méthode consiste à mesurer directement l'angle de poitrine avec un appareil spécial, dit "Anglemètre "; elle donne une idée du développement musculaire de part et d'autre du bréchet. A cet effet, le "West Virginia chicken breast meter " a été mis au point aux Etats-Unis : C'est une boîte plate d'oì sortent 2 mâchoires que l'on place près de l'extrémité antérieure du bréchet. On lit directement la valeur de l'angle sur un cadran gradué de 60 à Ioo degrés.

La méthode de l'anglemètre est simple, rapide et relativement précise d'où son utilisation dans de nombreuses stations américaines. Elle s'applique aussi bien sur le poulet vivant que sur les carcasses. Ia répétabilité de la mesure sur un même poulet est aussi bonne qu'avec le fil souple, d'après Colıins, Briss et ScotT, I950. Par contre, des essais réalisés à 1'Université de Virginie ont montré que les résultats varient avec les opérateurs et le type d'appareil. Il importe donc de garder le même opérateur et le même appareil pour mesurer les poulets d'un lot entier et de caractériser l'animal d'après son rang dans le lot, par exemple selon la méthode des probits proposée par Cochez et PERo, I954.

\section{L'ANGLEMĖTRE, AP- 8}

Les 2 photos ci-jointes montrent un appareil basé sur le principe de l'anglemètre de Virginie Occidentale : l'anglemètre AP- 8.

L'appareil comprend une partie fixe en contre-plaqué, une aiguille mobile en aluminium et un rapporteur d'angle en matière plastique transparente, divisé en grades. L'aiguille est solidaire d'un fil élastique en caoutchouc qui donne une grande souplesse de mouvement. L'ensemble pèse environ 80 grammes ; il est très maniable.

Pour la mesure, le poulet est maintenu sur le dos, à plat sur une table surélevée. L'appareil est placé perpendiculairement au bréchet, en arrière de l'extrémité antérieure, de façon que la plaquette du bâti fixe repose bien à plat sur un côté de la poitrine et que le bréchet vienne toucher la butée axiale; la partie mobile reliée à l'aiguille s'appuie sur l'autre côté de la poitrine. On peut mesurer ainsi 300 poulets à l'heure avec un opérateur, 2 assistants et I listeur.

La précision de l'appareil a été déterminée sur 37 poulets vivants qui ont été mesurés 5 fois. L'erreur absolue moyenne a été de 0,70 grade et l'erreur relative moyenne de $r, 33$ p. Ioo. Les mêmes poulets ont été abattus et 1'angle mesuré 3 fois sur la carcasse. L'erreur relative moyenne a été de $\mathrm{I}, 02 \mathrm{p}$. Ioo et la corrélation entre la mesure sur le vivant et la mesure sur la carcasse : 0,02 . Cette précision nous parait satisfaisante eu égard à la difficulté de la mesure. 


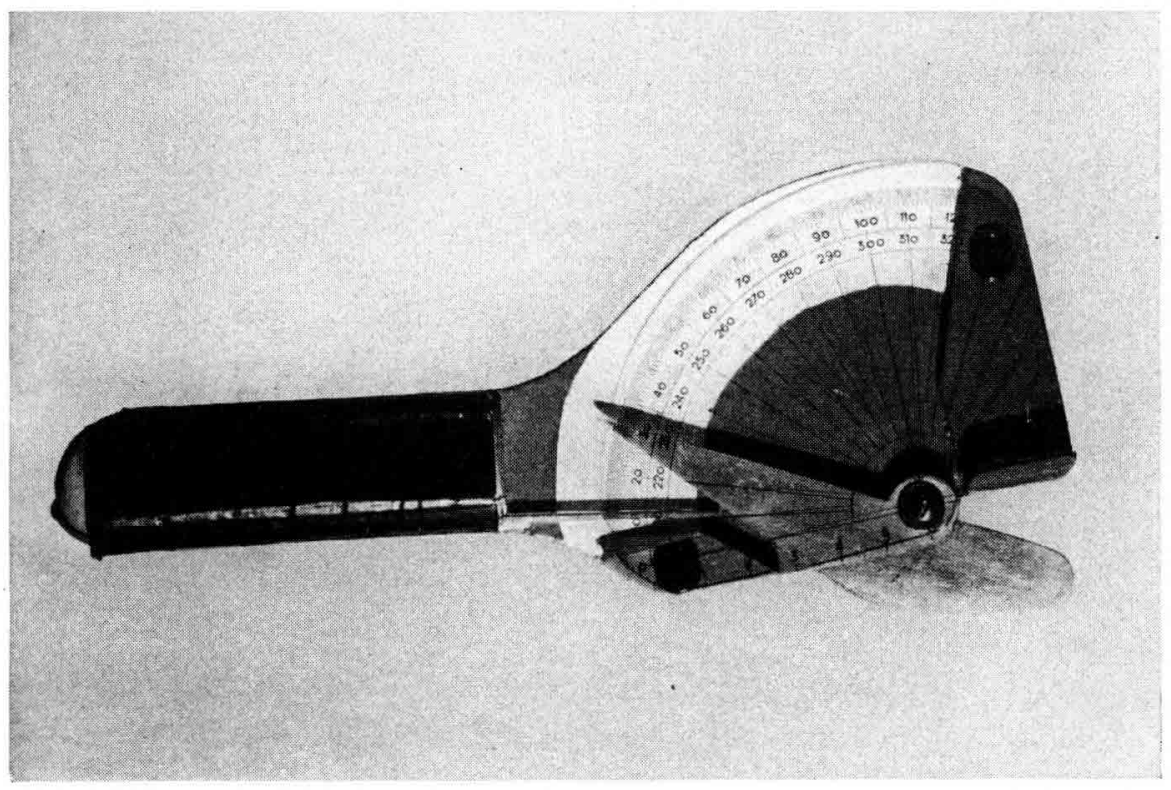

FIG. I. - Vue d'ensemble de l'Anglemètre AP.8

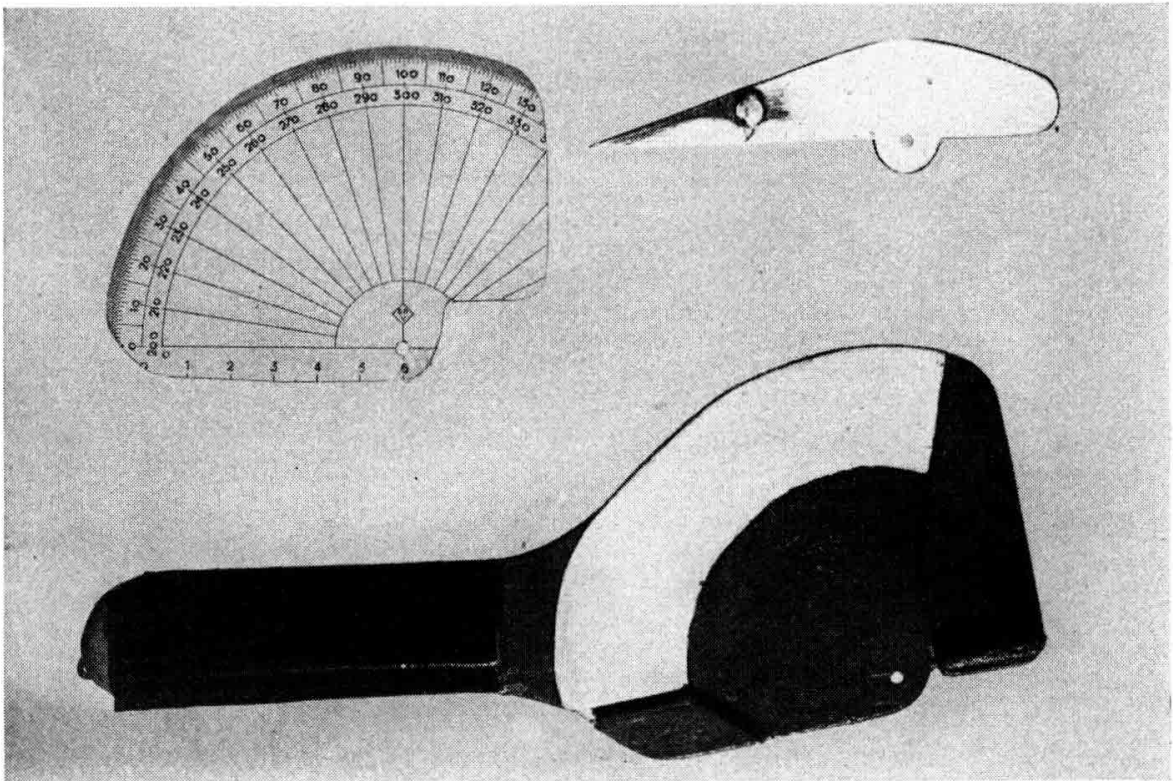

FIG. 2. - Les éléments constitutifs de l'Anglemètre AP-8. En bas, la partie fixe. En haut, et à droite vue inférieure de l'aiguille montrant le téton où s'accroche le brin élastique. En haut et à gauche le rapporteur d'angle divisé en grades. 
L'amplitude des variations entre les mesures à 8 semaines est de l'ordre de I5 à 25 grades. On obtient des chiffres faciles à manipuler. La répartition obtenue se rapproche d'une courbe normale où l'intervalle de classe - I grade -- représente 0,25 à 0,30 écart-type.

Reçu en décembre 1960.

\section{SUMMARY}

NOTE ON THE METHOD OF APPRAISING BREAST CONTOUR OF BROILERS

A short review is given of the 4 principals methods of appraising breast contour of broilers : subjective appreciation, use of vernier calipers, use of a wire solder or a lead tape, use of a breast measuring device. One of these, the AP- 8 anglemeter, is described. Its précision was found to be $1,3 \%$ as relative error on live birds and I \% on carcasses. 300 broilers can be measured in an hour.

Le Directeur Gérant : B. LACLAviÈRE.

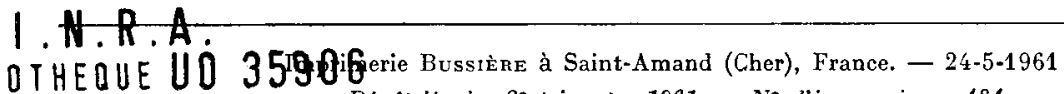

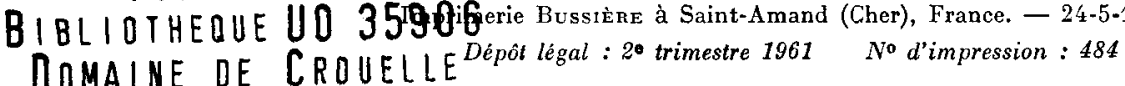

63039

CLERMONT-FD CEDEX? 